\title{
Cardiopatías de origen endocrino: una etiología frecuentemente olvidada
}

\section{Endocrine cardiomyopathies: a frequently forgotten etiology}

\author{
Laura Duque-González¹, Luis Miguel Ruiz-Velásquez², \\ José Luis Torres-Grajales ${ }^{3}$, Mauricio Duque-Ramírez ${ }^{4}$
}

\begin{abstract}
Resumen. Las enfermedades endocrinas comprenden un amplio espectro de trastornos hormonales de alta prevalencia, que reportan importantes tasas de morbimortalidad. Su asociación con desenlaces fuertes cardiovasculares, tales como infarto agudo de miocardio, enfermedad cerebrovascular e insuficiencia renal terminal, está claramente establecida; sin embargo, su relación con las cardiopatías es frecuentemente desestimada por el personal médico. Las alteraciones hipofisarias, en particular la acromegalia, el hipertiroidismo, el hipotiroidismo y las alteraciones suprarrenales, como son la enfermedad de Cushing, el hiperaldosteronismo y el feocromocitoma, están asociadas con la aparición de insuficiencia cardíaca, cardiopatía dilatada, arritmias cardíacas, e incluso, muerte súbita. La siguiente revisión pretende explicar de forma clara y precisa las principales cardiopatías de origen endocrinológico.
\end{abstract}

Palabras clave: cardiopatía, insuficiencia cardíaca, acromegalia, enfermedad de Cushing, hipotiroidismo, hiperparatiroidismo.

Abstract. Endocrine diseases comprise a broad spectrum of high prevalence hormonal disorders that report significant morbidity and mortality rates. Its association with strong cardiovascular outcomes such as acute myocardial infarction, cerebrovascular disease and end-stage renal failure are clearly established; however, its relation to heart disease is frequently underestimated by medical personnel. Pituitary disorders, particularly acromegaly, hyperthyroidism, hypothyroidism and adrenal di-

\footnotetext{
${ }^{1}$ Médica, Especialista en Medicina Interna, Residente de Cardiología. Universidad CES. Medellín, Colombia.

${ }^{2}$ Médico, Especialista en Medicina Interna y Epidemiología. Residente de Cardiología, Universidad CES. Medellín, Colombia.

E-mail: luismiguelruizvelasquez@gmail.com.

${ }^{3}$ Médico, Especialista en Medicina Interna y Endocrinología. Clínica Las Américas. Medellín, Colombia.

${ }^{4}$ Médico, Especialista en Medicina Interna, Cardiología y Electrofisiología. CES Cardiología, Universidad CES. Medellín,

Colombia.

Conflicto de interés: los autores declaran que no tienen conflicto de interés.

Medicina \& Laboratorio 2020;24:207-220. https://doi.org/10.36384/01232576.298

Recibido el 19 de septiembre de 2019; aceptado el 19 de noviembre de 2019. Editora Médica Colombiana S. A., 2020.
} 
sorders, such as Cushing's syndrome, hyperaldosteronism and pheochromocytoma, are associated with the appearance of heart failure, dilated heart disease, cardiac arrhythmias and even sudden death. The following review aims to clearly and precisely describe the main heart conditions associated with endocrine disorders.

Keywords: Cardiomyopathy, heart failure, acromegaly, Cushing's syndrome, hypothyroidism, hyperparathyroidism.

\section{Introducción}

La falla cardíaca es una enfermedad con una prevalencia estimada a nivel mundial de más de 37,7 millones de personas [1]; en Latinoamérica se estima una incidencia de 199 casos/100.000 personas-años [2], y en Colombia su prevalencia estimada es del 2,3\% de la población general [3].

Entre las etiologías más reconocidas en Latinoamérica se encuentran, en orden de frecuencia, las de origen isquémico, la cardiomiopatía dilatada idiopática, la hipertensiva, la enfermedad de Chagas, las de etiología valvular, alcohólica o asociada a tóxicos, periparto y las asociadas a miocarditis [4].

Existe una clara asociación entre los fenotipos metabólicos poco saludables, el sobrepeso y la obesidad, con el desarrollo de enfermedades cardiovasculares [5], y se tienen siempre presentes las patologías de origen endocrinológico entre los clásicos factores de riesgo para desarrollar falla cardíaca, pero estas son frecuentemente olvidadas como propias causantes de cardiopatías [4]. En esta revisión se tratará de resaltar la importante causalidad entre las enfermedades endocrinas y el desarrollo de alteraciones a nivel cardiovascular, teniendo en cuenta que no solo el incremento en la producción de hormonas conduce a enfermedad, sino también la falta de producción de las mismas [6].

\section{Fisiopatología de las cardiopatías de origen endocrino}

Las enfermedades de las glándulas endocrinas producen un sinnúmero de alteraciones cardíacas que pueden ser explicadas por tres mecanismos fisiopatológicos esenciales. El primer mecanismo es el producido secundario a una lesión directa del aparato cardiovascular, causada por la enfermedad primaria; el segundo y no menos importante, es el perpetuado por los factores de riesgo asociados a la enfermedad primaria, y, por último, el ocasionado por las modificaciones de la historia natural de una enfermedad cardiovascular, debidas a la endocrinopatía (tabla 1) [7].

El entender estos mecanismos fisiopatológicos y sus consecuencias, supone para el médico un reto clínico en la práctica diaria del paciente endocrinológico, y el inicio de un manejo oportuno ayudará a subsanar la alta tasa de morbi-mortalidad que acompaña a estas patologías [7].

Las alteraciones endocrinas que más a menudo se asocian con enfermedad cardiovascular serán explicadas en este artículo, con la excepción de la diabe- 
Tabla 1. Fisiopatología de la enfermedad cardiovascular asociada a endocrinopatías. Tomado y modificado de García Lled' JA. La afectación cardíaca en las enfermedades endocrinológicas. Rev Clin Esp 2000;200:566-569 [7]

\section{Lesión directa del aparato cardiovascular}

- Cardiomiopatía diabética

- Cardiomiopatía por hipertiroidismo

- Cardiomiopatía por hipotiroidismo

- Cardiomiopatía por hiperparatiroidismo

- Cardiomiopatía por hipoparatiroidismo

- Cardiomiopatía acromegálica

\section{Enfermedades endocrinas asociadas a factores de riesgo cardiovascular}

Hipertensión arterial asociada a:

- Diabetes mellitus

- Acromegalia

- Feocromocitoma

- Enfermedad de Cushing

- Hiperaldosteronismo

- Hipotiroidismo

- Hiperparatiroidismo

Dislipidemia asociada a:

- Diabetes mellitus

- Hipotiroidismo

- Enfermedad de Cushing

Diabetes mellitus asociada a:

- Acromegalia

- Obesidad

- Enfermedad de Cushing

Obesidad asociada a:

- Diabetes mellitus

- Acromegalia

- Enfermedad de Cushing

- Hiperprolactinemia

- Panhipopituitarismo

- Hiperandrogenismo

- Síndrome de ovario poliquístico

\section{Modificación de la evolución de la enfermedad cardiovascular debida a una endocrinopatía}

- Historia natural de la cardiopatía isquémica en un paciente con diabetes mellitus

- Riesgo trombogénico y evolución de la fibrilación auricular en un paciente con hipertiroidismo 
tes mellitus, que por su magnitud, merece una revisión por separado.

\section{Trastornos hormonales y cardiopatías asociadas}

Son diversos los desórdenes hormonales que pueden asociarse con alteraciones cardiovasculares. A continuación se describen los de mayor importancia, de acuerdo con la hormona implicada.

\section{Hormona de crecimiento}

El exceso de hormona de crecimiento (HC) conduce a acromegalia, una patología poco común que generalmente es producida por la hipersecreción de HC secundaria a un adenoma somatotropo [8]. La edad media de aparición es alrededor de los 30 años, pero su diagnóstico puede tardarse hasta los 40 ó 50 años, cuando las características físicas típicas son más evidentes. No se ha descrito en la literatura diferencias en la presentación según el sexo ni la raza $[9,10]$.

Para hacer el diagnóstico se debe tener una alta sospecha clínica, siendo la tamización el primer paso para llegar a este diagnóstico; esta se realiza con la medición de IGF-1 (del inglés, insulinlike growth factor 1). Para su valor de referencia no existe un punto de corte global, sino que se recomienda ajustarlo según el sexo y la edad [11]; si la primera prueba de tamización es positiva, se debe confirmar el diagnóstico con la medición de HC después de una carga oral de glucosa de $75 \mathrm{~g}$ [10].

Entre las manifestaciones clínicas más frecuentes de la acromegalia, en más del $60 \%$ de los pacientes, se encuentran el crecimiento acral, la deformidad facial con macroglosia, aumento del espacio interdental, prognatismo y malo- clusión, el edema de tejidos blandos y la hiperhidrosis; entre el $30 \%$ al $60 \%$ de los pacientes cursan con cefalea, disfunción eréctil, artritis, fatiga, parestesias y neuropatía periférica; finalmente, menos del 30\% manifiestan depresión, síndrome del túnel del carpo, somnolencia, miopatía, disnea, hipertricosis o galactorrea [12].

Más del $60 \%$ de los pacientes con acromegalia presentan alteraciones cardiovasculares [9]. La hipertrofia ventricular está presente en el 65,1\% de los pacientes con acromegalia, la disfunción diastólica en el 50,5\% y la disfunción sistólica en el 19,6\%; esta población tiene un riesgo aumentado $(\mathrm{OR}=13,1-28,2)$ de desarrollar este tipo de complicaciones, en comparación con la población general [13]. Las complicaciones cardíacas asociadas a la acromegalia se definen como cardiomiopatía acromegálica, e incluyen la alteración en la composición del miocardio a nivel de los miocitos e intercelular, alteración en la función sistólica y diastólica, enfermedad valvular con insuficiencia mitral y aórtica, y alteraciones en la conducción [14].

La estructura y crecimiento de los miocitos se ven afectados por el aumento en la captación de aminoácidos, el aumento de la expresión de genes musculares cardíacos (troponina 1, cadena ligera miosina-2, $\alpha$ actina y proteína fijadora de (GF-1) y la inhibición de apoptosis de los miocitos, causando un incremento en el tamaño del miocito, sarcomerogénesis y prevención de la pérdida de los mismos; la $\mathrm{HC}$ aumenta el depósito de colágeno cardíaco y el IGF-1 promueve la síntesis de colágeno por los fibroblastos, llevando a fibrosis cardíaca, comprometiendo inicialmente la función diastólica y por último, la sistólica [14]. 
El estímulo constante de la $\mathrm{HC}$ y el IGF-1, aumentan la expresión de genes contráctiles, lo cual conduce a un incremento significativo a la sensibilidad al calcio por parte de los miofilamentos y a mayor disponibilidad de calcio a nivel intracelular, facilitando la aparición de hipertrofia concéntrica, debido al ensamblaje de nuevos sarcómeros en paralelo. Esta hipertrofia puede comprometer ambos ventrículos, pero es más significativa en el ventrículo izquierdo y en el septo interventricular [14]; vale la pena aclarar que todo lo anteriormente mencionado se potencia con la hipertensión crónica causada por la misma acromegalia, la cual está presente en más del $50 \%$ de la población, siendo la hipertensión la etiología más fácil de identificar como causante de la cardiopatía [15].

Con base en lo anterior, se pueden plantear tres estadios de la enfermedad cardiovascular:

- Fase inicial: se produce con el inicio de la acromegalia, caracterizada por hipertrofia concéntrica biventricular, aumento en la contractilidad miocárdica, salida sistólica y aumento de la frecuencia cardíaca, comportándose como un síndrome hiperdinámico.

- Segunda fase: aumento de la hipertrofia ventricular y disminución del llenado diastólico secundario a fibrosis, con disminución del tiempo de eyección del ventrículo izquierdo y de la fracción de eyección ventricular izquierda (FEVI) durante el ejercicio.

- Fase terminal: disfunción diastólica y sistólica en reposo, y bajo gasto cardíaco $[15,16]$.
Desafortunadamente, la mayoría de los pacientes se diagnostican en la fase terminal de la cardiomiopatía acromegálica, cuando la disfunción sistólica se superpone al depósito de colágeno extracelular y hay trastorno miofibrilar e infiltración linfo-mononuclear que produce amplias áreas de necrosis, Ilevando a un deterioro de la arquitectura cardíaca con progresión a insuficiencia cardíaca congestiva $[17,18]$.

Entre los factores de riesgo para desarrollar la cardiomiopatía acromegálica se encuentran la edad mayor de 50 años, la presencia de hipertensión arterial, niveles altos de HC e IGF-1, y una duración de la enfermedad mayor de 10 años [16].

El tratamiento para la acromegalia, dirigido hacia el control del exceso de $\mathrm{HC}$ e IGF-1, podría alterar la progresión de la cardiomiopatía acromegálica [15], modificando el sombrío pronóstico de la enfermedad, y disminuyendo el riesgo de mortalidad general, que ha sido estimado hasta en 1,7 veces mayor, comparado con el de la población general [14].

De otra parte, la deficiencia de $\mathrm{HC}$ es más prevalente en niños y adolescentes, y puede asociarse con una reducción del grosor y masa miocárdica, y con disminución importante de la tolerancia al ejercicio [6].

\section{Cortisol}

La hormona corticotrópica (ACTH), liberada por la adenohipófisis, tiene una acción directa sobre la zona fasciculada de la corteza suprarrenal, estimulando así la producción de cortisol [19]. El exceso de cortisol es llamado síndrome de Cushing, ya sea por el exceso endógeno o exógeno de glucocorticoides [20]. 
El diagnóstico de síndrome de $\mathrm{Cu}$ shing se realiza demostrando evidencia inequívoca de hipercortisolismo autónomo, generalmente a través de la combinación de pruebas iniciales y estudios confirmatorios [21]. Para evaluar la presencia de hipercortisolismo se utilizan varios enfoques [21-23]:

1. Evaluar la excreción urinaria de cortisol en un período de 24 horas.

2. Documentar la pérdida de la inhibición por retroalimentación del cortisol en el eje hipotálamo-hipófisis-adrenal, con prueba de supresión con dosis de $1 \mathrm{mg}$ de dexametasona, para medir el nivel de cortisol al día siguiente, a las 8 a.m.

3. Documentar la pérdida del ritmo circadiano en la secreción de cortisol, con la medición de cortisol salival a la media noche.

4. Prueba de freno con dosis bajas de dexametasona, administrando dosis de 0,5 mg cada 6 horas por 48 horas, y midiendo el nivel de cortisol al finalizar este período de tiempo.

Por lo general, se puede comenzar con cualquiera de los enfoques mencionados anteriormente, pero se requiere la combinación de 2 de las 3 pruebas para confirmar el diagnóstico [24]. La medida de la cortisoluria en orina de 24 horas es la prueba estándar de oro para diagnosticar el síndrome de $\mathrm{Cu}$ shing; un aumento de 3 a 4 veces el límite superior de lo normal, es diagnóstico de síndrome de Cushing $[21,25]$.

Después de que el hipercortisolismo está firmemente establecido, la causa debe explorarse más a fondo para determinar si es suprarrenal, hipofisaria o por producción ectópica de ACTH
$[21,26]$. Las mediciones de las concentraciones plasmáticas de $\mathrm{ACTH}$, que deberían medirse en forma simultánea al cortisol basal, antes de las pruebas dinámicas, son las que diferencian el síndrome de Cushing ACTH dependiente $\mathrm{O}$ independiente. Los niveles de ACTH bajos o indetectables sugieren neoplasia suprarrenal como causa del hipercortisolismo, en tanto que las concentraciones normales, no esperadas, o elevadas de ACTH, sugieren un adenoma hipofisario o fuente de producción ectópica de ACTH (usualmente con valores más elevados de corticotropina) $[20,23,27]$.

Según la clasificación del síndrome de Cushing, se define la prueba de localización con imágenes. Se puede utilizar la tomografía o resonancia de glándulas suprarrenales en el síndrome de Cushing ACTH independiente. Si se sospecha adenoma hipofisario, se puede realizar imagen por resonancia magnética de silla turca, $y$, finalmente, si hay sospecha de producción ectópica de ACTH, se puede hacer tomografía de tórax y abdomen, resonancia de abdomen o gammagrafía con radiofármaco y receptores de somatostatina (octreoscan) $[21,28]$.

Entre las manifestaciones más frecuentes del síndrome de Cushing se encuentran el aumento de peso, la facies de luna llena, la hipertensión arterial hasta en el $75 \%$ de los casos [19], las alteraciones en piel y anexos, incluyendo hirsutismo, equimosis y estrías, las alteraciones psiquiátricas como labilidad emocional, depresión, agitación, irritabilidad, ansiedad, ataques de pánico, paranoia e insomnio, la miopatía proximal, y la osteoporosis [20]. Estos pacientes tienen un riesgo cardiovascular hasta 5 veces mayor que el de la población sin esta patología [19]. 
La homocisteinemia, la dislipidemia que lleva a aterosclerosis acelerada y la hipertensión crónica derivada del aumento en la retención de sodio y agua [29], podrían explicar por si solas el origen de la cardiopatía; sin embargo, se ha encontrado que el exceso de cortisol conlleva cambios estructurales y funcionales del miocardio, tales como el remodelamiento concéntrico, la hipertrofia ventricular observada hasta en el $50 \%$ a $70 \%$ de los pacientes con síndrome de $\mathrm{Cu}$ shing, la fibrosis cardíaca, y por último y menos frecuente, la disfunción sistodiastólica, manifestada como síndrome de falla cardíaca [30].

Los pacientes con síndrome de Cushing tienen reducción en la fracción de eyección del ventrículo izquierdo, ventrículo derecho y aurícula izquierda, con un aumento de la masa del ventrículo izquierdo, evaluados por resonancia cardíaca. Después del tratamiento del hipercortisolismo se ha evidenciado una reversibilidad de los mismos [31].

Existen en la literatura más de 30 casos reportados de cardiopatía dilatada asociada a síndrome de Cushing, siendo el aspecto más importante a resaltar la reversibilidad de la patología cardíaca con la implementación del tratamiento dirigido [32]; todos los casos presentaron una reducción de la FEVI y demostraron una mejoría, hasta lograr valores de normalidad en este mismo parámetro en menos de un año después de instaurado el manejo [30,33]. Esto ha permitido comprobar que la gran culpable de la disfunción cardíaca es la secreción anormal de cortisol, ya que se ha encontrado que los otros factores de riesgo cardiovasculares, como la hipertensión y la dislipidemia, persisten a pesar del tratamiento médico óptimo [34].

\section{Hormona paratiroidea}

Las glándulas paratiroides son las encargadas de secretar hormona paratiroidea (PTH). Esta secreción es modulada por los niveles de calcio, fósforo y vitamina $D$ séricos; dicha hormona tiene tres blancos principales: el hueso en donde activa la resorción de calcio, el riñón en donde aumenta la absorción de calcio, disminuye la de fosfato y activa la vitamina D, y, por último, el intestino en donde aumenta la reabsorción de calcio e incrementa la transcripción de la enzima 1-alfa-hidroxilasa, que lleva a un aumento en la forma activa de la vitamina $D(1,25$-dihidroxivitamina D) [35].

El hiperparatiroidismo primario es el tercer trastorno endocrino más frecuente, con una mayor incidencia en mujeres posmenopáusicas; la causa principal de este trastorno es la presencia de adenomas paratiroideos en el $75 \%$ a $85 \%$ de los casos, seguidos de hiperplasia paratiroidea en el $15 \%$ de los casos, y de carcinoma de paratiroides en el $2 \%[36,37]$.

La hipertrofia ventricular izquierda es la patología más común observada en pacientes con hiperparatiroidismo, y es independiente de la hipertensión arterial, ya que la PTH actúa sobre el receptor de la PTH tipo 1 (PTH1R) a nivel cardíaco, el cual a través de la proteína $G$, desencadena una activación de la proteína quinasa $C$, aumentando el influjo de calcio a los miocitos y estimulando su proliferación e hipertrofia [35].

Los pacientes con niveles de PTH $\geq 65$ $\mathrm{pg} / \mathrm{mL}$ tienen un $44 \%$ más de riesgo de desarrollar falla cardíaca, la cual es secundaria a la hipertrofia de los miocitos y al aumento de la cronotropía, debido al alto influjo de calcio en los mismos 
[35]. Entre otras alteraciones cardiovasculares con las que cursa el hiperparatiroidismo se encuentran el acortamiento del intervalo QT [38], la hipertensión arterial y las valvulopatías secundarias a calcificación de las valvas, de predominio aórtico y mitral [39].

El tratamiento del exceso de PTH, como ocurre con la resección quirúrgica en adenomas paratiroideos, disminuye la hipertrofia ventricular izquierda en algunos pacientes, e incluso se ha observado una reducción significativa de las alteraciones del ritmo cardíaco y en la progresión de las calcificaciones valvulares $[36,40]$.

Al ser la vitamina $D$ uno de las grandes contribuyentes en la homeostasis de la PTH y el calcio, se ha tratado de investigar la causalidad entre la hipovitaminosis D y las alteraciones cardiovasculares, ya que además se han encontrado receptores de vitamina $D$ en los miocitos, el músculo vascular liso y el endotelio vascular, que interfieren con el transporte de calcio y el aumento de la hipertrofia, inflamación y fibrosis de los miocitos; esta correlación no ha podido ser confirmada en ensayos clínicos, pero la sospecha aún permanece [41].

Por otro lado, el hipoparatiroidismo es una entidad menos frecuente. En la mayoría de la población adulta su etiología es secundaria a una cirugía de resección de paratiroides [42]; en esta patología prima la hipocalcemia, la cual reduce la contractilidad cardíaca, llevando a disfunción sistólica, que en estados iniciales puede revertirse posterior al tratamiento de la misma. Otras alteraciones asociadas con el hipoparatiroidismo son la prolongación del intervalo QT, predisponiendo a la aparición de arritmias potencialmente mortales, y la cardiopatía dilatada [35].

\section{Hormonas tiroideas}

La glándula tiroides produce dos tipos de hormonas, la tiroxina (T4) y la triyodotironina (T3), siendo esta última la forma activa a nivel cardiovascular. La producción y secreción de estas hormonas está influenciada por múltiples factores, principalmente los niveles de la hormona estimulante de la tiroides (TSH) y los de la hormona liberadora de tirotropina (TRH) [43].

El exceso de hormona tiroidea tiene dos sitios de acción a nivel cardiovascular. Por una parte actúa directamente en el corazón produciendo taquicardia, aumento de la masa ventricular, disfunción diastólica, arritmias, cardiopatía dilatada y falla cardíaca [44], y por otra parte, su segundo sitio de acción es la vasculatura sistémica, con manifestaciones que incluyen contracción vascular, aumento del volumen circulante e hipertensión arterial pulmonar y sistémica [45].

Las alteraciones hemodinámicas asociadas al hipertiroidismo disminuyen la reserva contráctil del miocardio, imposibilitando el aumento en la FEVI y el gasto cardíaco durante estados de alta demanda, como ejercicio, taquiarritmias y sepsis, entre otros [40]. La falla cardíaca se presenta hasta en el $6 \%$ de los pacientes que cursan con hipertiroidismo; sin embargo, menos de $1 \%$ van a desarrollar cardiomiopatía dilatada con disfunción sistólica [46].

Los efectos tóxicos del exceso de hormona tiroidea conducen a una alteración de la producción energética de los miocitos, de su síntesis proteica y de la función contráctil de las miofibrillas, precipitando la aparición de insuficiencia cardíaca, incluso en pacientes sin ninguna cardiopatía previa o en ausencia de factores de riesgo cardiovasculares [47]. 
Se definen tres etapas de la cardiomiopatía tirotóxica:

1. Hipercinética: FEVI conservada, pero no aumenta con el esfuerzo.

2. Normocinética: etapa compensatoria, con hipertrofia miocárdica reversible y gasto cardíaco preservado.

3. Hipocinética: baja FEVI y bajo gasto cardíaco, con hipertrofia y dilatación [43].

Las alteraciones en el ritmo cardíaco son bien conocidas, siendo la más frecuente la taquicardia sinusal, hasta en el $60 \%$ de los pacientes, y las taquiarritmias supraventriculares, como la fibrilación auricular en el $11 \%$ [48].

Estos cambios a nivel cardiovascular pueden revertirse por completo; a pesar de esto, se han reportado factores de mal pronóstico para lograr reversibilidad, tales como la larga duración del hipertiroidismo sin tratamiento, mayor de 10 meses, los niveles altos de T3 y el no uso de beta bloqueadores, entre otros [45].

De otra parte, la deficiencia de hormonas tiroideas se asocia con una disminución del gasto cardíaco, la frecuencia cardíaca, el volumen sistólico y la contractilidad miocárdica, y con un aumento conside- rable de la resistencia vascular sistémica [49]. Por lo general, su presentación es menos evidente a lo mencionado en el hipertiroidismo, y las alteraciones a nivel cardiovascular ocurren cuando la instauración de la deficiencia hormonal es de larga data (tabla 2) [43,50].

Entre las manifestaciones cardíacas clásicas se encuentran la bradicardia sinusal, el derrame pericárdico y la insuficiencia cardíaca [51]. El hipotiroidismo, incluso al ser subclínico, conduce a una actividad reducida de la enzima ATPasa de $\mathrm{Ca}^{2+}$ del retículo sarcoplásmico (SERCA2), alterando el ciclo del calcio intracelular, aumentando la rigidez miocárdica y produciendo disfunción diastólica del ventrículo izquierdo $[52,53]$. Las alteraciones electrocardiográficas en el hipotiroidismo son la bradicardia sinusal, el bajo voltaje y la prolongación del intervalo QT, predisponiendo así a arritmias fatales como la torsade de pointes [52].

Si bien es frecuente encontrar niveles bajos de hormonas tiroideas en pacientes con falla cardíaca, entre $15 \%$ y $30 \%$, no todos los casos son causados por el propio hipotiroidismo, pero es de vital importancia recalcar que los pacientes con FEVI reducida y bajos niveles de T3, tienen mayor mortalidad que los pacientes con valores de FEVI similares

Tabla 2. Fenómenos fisiopatológicos de las alteraciones cardíacas en el hipotiroidismo

Disminución en la precarga: dada por el bajo volumen arterial y la disfunción diastólica

Aumento en la poscarga: ocasionada por resistencias vasculares sistémicas altas

Alteración en el cronotropismo: hay disminución en la expresión de T3 en las células cardíacas, sin lograr estimular las enzimas sodio-potasio ATPasa y la calcio ATPasa en el retículo endoplásmico

Alteraciones del ritmo (bradicardia) y derrame pericárdico: pueden empeorar y perpetuar la cardiopatía 
y concentraciones de T3 normales; por lo tanto, la búsqueda del hipotiroidismo en pacientes con falla cardíaca no solo es importante para descartar etiologías reversibles como el hipotiroidismo, sino también para reducir la mortalidad asociada a etiologías diferentes a este [54].

\section{Otras causas hormonales}

Existen otras patologías de origen endocrino que pudiesen asociarse con alteraciones en la función cardíaca, entre estas cabe resaltar las siguientes:

- Hiperaldosteronismo: el exceso de aldosterona causa una remodelación cardíaca mal-adaptativa, asociándose a hipertrofia ventricular izquierda (por estimulación sobre el crecimiento e hipertrofia de los miocitos), disfunción diastólica, fibrosis cardíaca (asociada a la deposición de colágeno, activación de células inflamatorias y proliferación de fibroblastos), y disminuye la biodisponibilidad del óxido nítrico, lo cual inhibe la relajación endotelial [6]; es importante resaltar que el aumento en la masa ventricular izquierda es desproporcionado al compararse con la hipertrofia causada por hipertensión arterial esencial [55].

- Feocromocitoma: los tumores productores de catecolaminas pueden causar cardiomiopatía hasta en el $26 \%$ de los casos, isquemia miocárdica o aturdimiento miocárdico debido a vasoespasmo coronario, y shock cardiogénico agudo secundario a síndrome de Tako-tsubo. Las cardiopatías pueden ser dilatadas, con contractilidad y FEVI disminuidas, o hipertróficas; estas últimas son principalmente debidas a la hipertensión arterial, más que al propio exceso de catecolaminas [55].
- Deficiencia de andrógenos: hasta el $40 \%$ de los pacientes con falla cardíaca presenta una disminución en los niveles de testosterona total y libre, lo cual tiene un papel importante en la vasodilatación coronaria mediada por óxido nítrico, a través de la inducción de canales de calcio y potasio. Anteriormente, el uso de terapia de reemplazo hormonal en estos pacientes estaba contraindicado por el efecto en la retención hídrica, pero se demostró que los beneficios superaban los riesgos al reducir la resistencia vascular sistémica y la poscarga, y mejorar el gasto cardíaco [56].

\section{Conclusión}

Es de vital importancia indagar a fondo la etiología de las cardiopatías, ya que muchas veces son clasificadas como de origen hipertensivo, isquémico o arrítmico, o pertenecientes al gran grupo de las cardiopatías dilatadas idiopáticas; siendo las tres primeras posibles manifestaciones en común de etiologías de origen endocrinológico, y no las causantes de la misma cardiopatía. En la figura 1 se resumen las etiologías descritas y sus manifestaciones cardiovasculares más frecuentes; por último, en el grupo de las cardiopatías dilatadas idiopáticas, las características clínicas del paciente y el desarrollo de un enfoque diagnóstico integral, pueden llevar al médico a encontrar desencadenantes endocrinológicos que tengan un impacto en la progresión de la enfermedad.

\section{Referencias}

1. Ziaeian B, Fonarow GC. Epidemiology and aetiology of heart failure. Nat Rev Cardiol 2016;13:368-378. https://doi.org/10.1038/nrcardio.2016.25. 


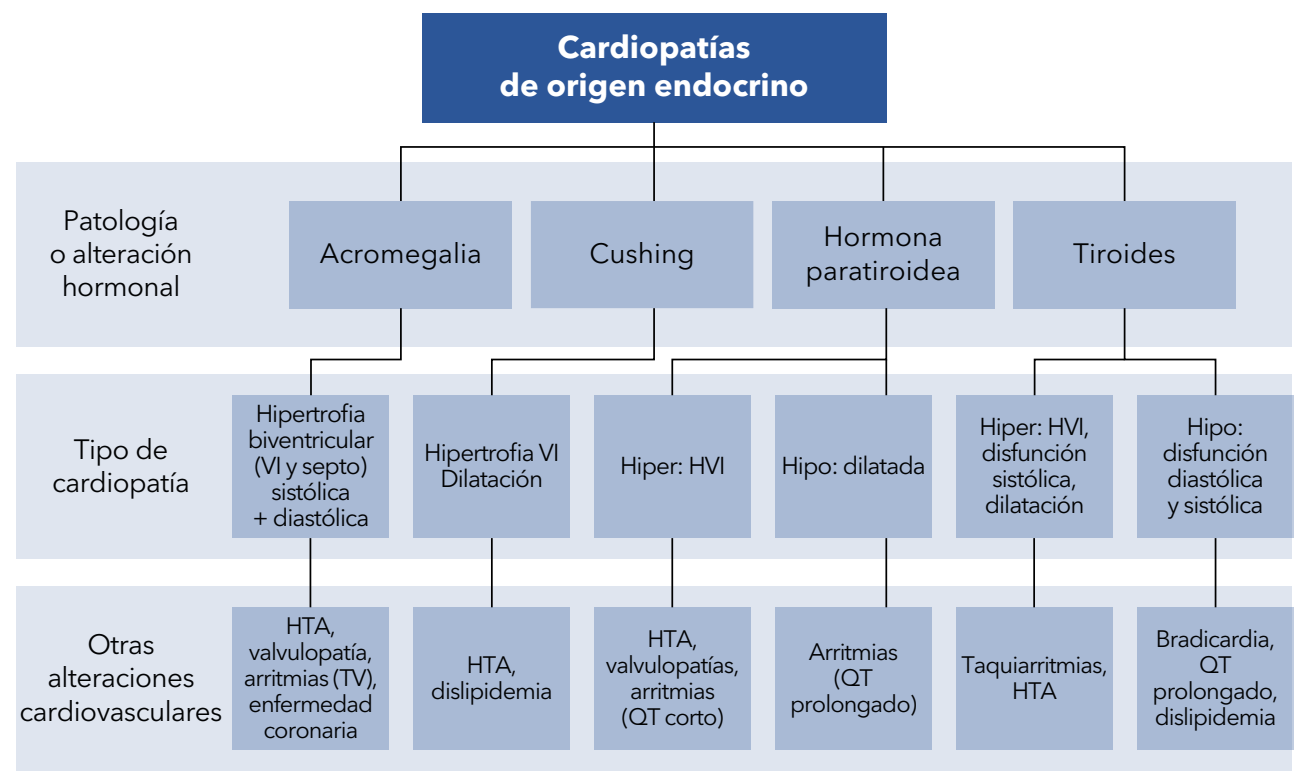

Figura 1. Resumen de las etiologías descritas, con el tipo de presentación de cardiopatía y otras manifestaciones cardiovasculares con las que pueden cursar. VI: ventrículo izquierdo; HVI: hipertrofia ventricular izquierda; HTA: hipertensión arterial; TV: taquicardia ventricular.

2. Ciapponi A, Alcaraz A, Calderón M, Matta MG, Chaparro M, Soto $\mathbf{N}$, et al. Carga de enfermedad de la insuficiencia cardiaca en América Latina: revisión sistemática y metanálisis. Rev Esp Cardiol 2016;69:1051-1060. https://doi.org/https://doi.org/10.1016/j.recesp.2016.04.045.

3. Gómez E. Capítulo 2. Introducción, epidemiología de la falla cardiaca e historia de las clínicas de falla cardiaca en Colombia. Rev Colomb Cardiol 2016;23:6-12. https://doi.org/https:// doi.org/10.1016/j.rccar.2016.01.004.

4. Bocchi EA, Arias A, Verdejo H, Diez M, Gomez E, Castro $\mathbf{P}$, et al. The reality of heart failure in Latin America. J Am Coll Cardiol 2013;62:949-958. https://doi.org/10.1016/j. jacc.2013.06.013.

5. Mirzababaei A, Djafarian K, Mozafari H, ShabBidar S. The long-term prognosis of heart diseases for different metabolic phenotypes: a systematic review and meta-analysis of prospective cohort studies. Endocrine 2019;63:439-462. https://doi.org/10.1007/s12020-019-01840-0.
6. Rhee SS, Pearce EN. The endocrine system and the heart: A review. Rev Esp Cardiol 2011;64:220231. https://doi.org/10.1016/j.rec.2010.10.016

7. García-Lled' JA. La afectación cardíaca en las enfermedades endocrinológicas. Rev Clin Esp 2000;200:566-569. https://doi.org/https://doi. org/10.1016/S0014-2565(00)70721-1.

8. Colao A, Grasso LFS, Giustina A, Melmed S, Chanson P, Pereira AM, et al. Acromegaly. Nat Rev Dis Primers 2019;5:20. https://doi. org/10.1038/s41572-019-0071-6.

9. AIDallal S. Acromegaly: a challenging condition to diagnose. Int J Gen Med 2018;11:337343. https://doi.org/10.2147/IJGM.S169611.

10. Zahr R, Fleseriu M. Updates in diagnosis and treatment of acromegaly. Eur Endocrinol 2018;14:57-61. https://doi.org/10.17925/ EE.2018.14.2.57.

11. Melmed S, Bronstein MD, Chanson $P$, Klibanski A, Casanueva FF, Wass JAH, et al. A consensus statement on acromegaly therapeutic outcomes. Nat Rev Endocrinol 2018;14:552-561. https://doi.org/10.1038/s41574-018-0058-5. 
12. Vilar L, Vilar CF, Lyra $R$, Lyra $R$, Naves LA. Acromegaly: clinical features at diagnosis. Pituitary 2017;20:22-32. https://doi.org/10.1007/ s11102-016-0772-8.

13. Guo $X$, Fu H, Pang $H$, Xing B. Risk of left ventricular hypertrophy and diastolic and systolic dysfunction in acromegaly: A meta-analysis. J Clin Neurosci 2018;48:28-33. https://doi. org/10.1016/j.jocn.2017.10.067.

14. Sharma AN, Tan $M$, Amsterdam EA, Singh GD. Acromegalic cardiomyopathy: Epidemiology, diagnosis, and management. Clinical cardiology 2018;41:419-425. https://doi. org/10.1002/clc.22867.

15. Ramos-Levi AM, Marazuela M. Cardiovascular comorbidities in acromegaly: an update on their diagnosis and management. Endocrine 2017;55:346-359. https://doi.org/10.1007/ s12020-016-1191-3.

16. Goldberg MD, Vadera N, Yandrapalli S, Frishman WH. Acromegalic cardiomyopathy: An overview of risk factors, clinical manifestations, and therapeutic options. Cardiol Rev 2018;26:307-311. https://doi.org/10.1097/ CRD.0000000000000215.

17. Mantri NM, Amsterdam E, Tan M, Singh GD. Power failure: Acromegalic cardiomyopathy. Am J Med 2016;129:674-677. https://doi. org/10.1016/j.amjmed.2016.02.041.

18. Sharma MD, Nguyen AV, Brown S, Robbins RJ. Cardiovascular disease in acromegaly. Methodist Debakey Cardiovasc J 2017;13:64-67. https:// doi.org/10.14797/mdcj-13-2-64.

19. Gutiérrez-Restrepo J, Sierra-Latorre G, Campuzano-Maya G. Síndrome de Cushing. Med Lab 2009;15:411-430.

20. Pappachan JM, Hariman C, Edavalath M, Waldron J, Hanna FW. Cushing's syndrome: a practical approach to diagnosis and differential diagnoses. J Clin Pathol 2017;70:350-359. https://doi. org/10.1136/jclinpath-2016-203933.

21. Bansal V, El Asmar N, Selman WR, Arafah BM. Pitfalls in the diagnosis and management of Cushing's syndrome. Neurosurg Focus 2015;38:E4. https://doi.org/10.3171/2014.11. Focus 14704.

22. Findling JW, Raff $\mathbf{H}$, Aron DC. The low-dose dexamethasone suppression test: a reevaluation in patients with Cushing's syndrome. J Clin Endocrinol Metab 2004;89:1222-1226. https://doi. org/10.1210/jc.2003-030207.

23. Nieman LK, Biller BM, Findling JW, NewellPrice J, Savage MO, Stewart PM, et al. The diagnosis of Cushing's syndrome: an Endocrine Society Clinical Practice Guideline. J Clin Endocrinol Metab 2008;93:1526-1540. https://doi. org/10.1210/jc.2008-0125.

24. Elamin MB, Murad MH, Mullan R, Erickson D, Harris K, Nadeem S, et al. Accuracy of diagnostic tests for Cushing's syndrome: a systematic review and metaanalyses. J Clin Endocrinol Metab 2008;93:1553-1562. https://doi.org/10.1210/ jc.2008-0139.

25. Nieman LK. Recent updates on the diagnosis and management of Cushing's syndrome. Endocrinol Metab (Seoul) 2018;33:139-146. https:// doi.org/10.3803/EnM.2018.33.2.139.

26. Loriaux DL. Diagnosis and differential diag nosis of Cushing's syndrome. N Engl J Med 2017;376:1451-1459. https://doi.org/10.1056/ NEJMra1505550.

27. Nieman LK. Diagnosis of Cushing's syndrome in the modern era. Endocrinol Metab Clin North Am 2018;47:259-273. https://doi.org/10.1016/j. ecl.2018.02.001.

28. Biller BM, Grossman AB, Stewart PM, Melmed S, Bertagna X, Bertherat J, et al. Treatment of adrenocorticotropin-dependent Cushing's syndrome: a consensus statement. J Clin Endocrinol Metab 2008;93:2454-2462. https://doi. org/10.1210/jc.2007-2734.

29. Whitworth JA, Mangos GJ, Kelly JJ. Cushing, cortisol, and cardiovascular disease. Hypertension 2000;36:912-916. https://doi.org/10.1161/01. hyp.36.5.912.

30. Shibusawa N, Yamada M, Hashida T, Hashimoto K, Satoh T, Horiguchi J, et al. Dilated cardiomyopathy as a presenting feature of Cushing's syndrome. Intern Med 2013;52:1067-1071. https:// doi.org/10.2169/internalmedicine.52.9051.

31. Kamenicky P, Redheuil A, Roux C, Salenave S, Kachenoura N, Raissouni Z, et al. Cardiac structure and function in Cushing's syndrome: a cardiac magnetic resonance imaging study. J Clin Endocrinol Metab 2014;99:E2144-2153. https:// doi.org/10.1210/jc.2014-1783. 
32. Aydogan BI, Gerede DM, Canpolat AG, Erdogan MF. Cushing's disease presented by reversible dilated cardiomyopathy. Case Rep Cardiol 2015;2015:980897. https://doi. org/10.1155/2015/980897.

33. Marchand L, Segrestin B, Lapoirie M, Favrel V, Dementhon J, Jouanneau E, et al. Dilated cardiomyopathy revealing Cushing disease: A case report and literature review. Medicine (Baltimore) 2015;94:e2011. https://doi.org/10.1097/ MD.0000000000002011.

34. De Leo M, Pivonello R, Auriemma RS, Cozzolino A, Vitale P, Simeoli C, et al. Cardiovascular disease in Cushing's syndrome: heart versus vasculature. Neuroendocrinology 2010;92 Suppl 1:50-54. https://doi.org/10.1159/000318566.

35. Brown SJ, Ruppe MD, Tabatabai LS. The parathyroid gland and heart disease. Methodist Debakey Cardiovasc J 2017;13:49-54. https:// doi.org/10.14797/mdcj-13-2-49.

36. Fraser WD. Hyperparathyroidism. Lancet 2009;374:145-158. https://doi.org/10.1016/ S0140-6736(09)60507-9.

37. Walker MD, Silverberg SJ. Primary hyperparathyroidism. Nat Rev Endocrinol 2018;14:115125. https://doi.org/10.1038/nrendo.2017.104.

38. Guimaraes $T$, Nobre-Menezes $M$, Cruz $D$, do Vale S, Bordalo A, Veiga A, et al. Hypercalcemic crisis and primary hyperparathyroidism: Cause of an unusual electrical storm. Rev Port Cardiol 2017;36:959 e951-959 e955. https://doi. org/10.1016/j.repc.2016.12.015.

39. Dokupilova A, Payer J. 24-hour outpatient ECG as a screening method in patients with primary hyperparathyroidism. Bratisl Lek Listy 2016;117:495500. https://doi.org/10.4149/bll_2016_096.

40. Pepe J, Cipriani $C$, Curione M, Biamonte F, Colangelo $\mathbf{L}$, Danese $\mathbf{V}$, et al. Reduction of arrhythmias in primary hyperparathyroidism, by parathyroidectomy, evaluated with 24-h ECG monitoring. Eur J Endocrinol 2018;179:117-124. https://doi.org/10.1530/EJE-17-0948.

41. Skaaby $\mathbf{T}$, Thuesen BH, Linneberg A. Vitamin $D$, cardiovascular disease and risk factors. Adv Exp Med Biol 2017;996:221-230. https://doi. org/10.1007/978-3-319-56017-5_18.

42. Mannstadt M, Bilezikian JP, Thakker RV, Hannan FM, Clarke BL, Rejnmark L, et al. Hypopa- rathyroidism. Nat Rev Dis Primers 2017;3:17055. https://doi.org/10.1038/nrdp.2017.55.

43. Vargas-Uricoechea $H$, Bonelo-Perdomo A, Sierra-Torres $\mathbf{C H}$. Effects of thyroid hormones on the heart. Clin Investig Arterioscler 2014;26:296-309. https://doi.org/10.1016/j.arteri.2014.07.003.

44. Ademolu A. Thyrotoxic cardiomyopathy: A study of three cases. Gerontol Geriatr Stud 2017;1:46-49. https://doi.org/10.31031/ GGS.2017.01.000512.

45. Oliveros-Ruiz L VM, Diez-Canseco LF, Cárdenas M, Hermosillo JAG,. Determinants of thyrotoxic cardiomyopathy recovery. BioMed Res Int 2013;2013:1-7. https://doi. org/10.1155/2013/452709

46. Al-Ghamdi AS, Aljohani N. Graves' thyrotoxicosis-induced reversible cardiomyopathy: a case report. Clin Med Insights Case Rep 2013;6:47-50. https://doi.org/10.4137/CCRep.S10534.

47. Osuna PM, Udovcic M, Sharma MD. Hyperthyroidism and the heart. Methodist Debakey Cardiovasc J 2017;13:60-63. https://doi. org/10.14797/mdcj-13-2-60.

48. Baladi IH, Rai AA, Ahmed SM. ECG changes in patients with primary hyperthyroidism. Pan Afr Med J 2018;30:246. https://doi.org/10.11604/ pamj.2018.30.246.12244.

49. Udovcic M, Pena RH, Patham B, Tabatabai L, Kansara A. Hypothyroidism and the heart. Methodist Debakey Cardiovasc J 2017;13:55-59. https://doi.org/10.14797/mdcj-13-2-55.

50. Vargas-Uricoechea $H$, Bonelo-Perdomo A. Thyroid dysfunction and heart failure: Mechanisms and associations. Curr Heart Fail Rep 2017;14:48-58. https://doi.org/10.1007/s11897017-0312-5.

51. Toft AD, Boon NA. Thyroid disease and the heart. Heart 2000;84:455-460. https://doi.org/10.1136/ heart.84.4.455.

52. Klein I, Danzi S. Thyroid disease and the heart. Curr Probl Cardiol 2016;41:65-92. https://doi. org/10.1016/j.cpcardiol.2015.04.002.

53. Bielecka-Dabrowa A, Godoy B, Suzuki T, Banach M, von Haehling S. Subclinical hypothyroidism and the development of heart failure: an overview of risk and effects on cardiac function. Clin Res Cardiol 2019;108:225-233. https://doi. org/10.1007/s00392-018-1340-1. 
54. Razvi S, Jabbar A, Pingitore A, Danzi S, Biondi B, Klein I, et al. Thyroid hormones and cardiovascular function and diseases. J Am Coll Cardiol 2018;71:1781-1796. https://doi.org/10.1016/j. jacc.2018.02.045

55. Binu A, Cherian K, Kapoor N, Chacko S, George $\mathbf{O}$, Paul T. The heart of the matter: Cardiac manifestations of endocrine disease. Indian J Endocrinol Metab 2017;21:919-925. https://doi. org/10.4103/ijem.IJEM_212_17.

56. Botero-Arango A, Duque-Ramírez M, DuqueGonzález L. Deficiencia de andrógenos y enfermedad cardiovascular en el hombre. Med Lab 2014;20:43-56 